\title{
Next-gen sequencing of multi-drug resistant Acinetobacter baumannii to determine antibiotic resistance genotypes
}

\author{
Leon Dent ${ }^{1 *}$, Dana Marshall ${ }^{1}$, Robert Hulette ${ }^{2}$, Siddharth Pratap ${ }^{3}$ \\ From UT-ORNL-KBRIN Bioinformatics Summit 2010 \\ Cadiz, KY, USA. 19-21 March 2010
}

\section{Background}

Multi-drug resistant (MDR) Acinetobacter baumannii is an important cause of hospital acquired infection and often increases mortality and length of stay[1-3]. The mechanisms of resistance include: (1) antimicrobialinactivating enzymes such as $\square$-lactamases, (2) alteration of membrane porin channels, and (3) mutations that change cellular functions [4]. Accurate genotyping and correlation to antimicrobial susceptibility will help prevent and treat outbreaks of Acinetobacter.

The genome of A. baumannii ranges from 3.2 Megabases $(\mathrm{Mb})$ in the drug sensitive SDF strain up to 3.9 $\mathrm{Mb}$ in the MDR AYE strain. A surprisingly high proportion of baumannii ORFs, (15\%-20\%), are located in resistance islands or "alien islands" - long stretches of DNA acquired from a foreign source. The MDR AYE strain has an $86 \mathrm{~Kb}$ island containing $45-50$ drug resistance genes located in an insertion hotspot [5]. Our study aims to sequence several A. baumannii isolates from Metro Nashville General (NGH) Hospital and conduct a strain-to-reference genomic characterization of clinical virulence factors.

\section{Materials and methods}

A retrospective review of the NGH hospital epidemiology data base included 247 isolates of $A$. baumannii from 164 patients (submitted, BMC Infectious Disease). Cluster Software version 2.11 and TreeView software grouped resistance phenotypes into six categories (see Figure 1) [6].

1.Pan resistant

2.Pan sensitive

\footnotetext{
* Correspondence: Ident@mmc.edu

'Department of Surgery, Meharry Medical College, Nashville, TN 37208, USA
}

3.Sensitive to meropenem /imipenem only.

4.Sensitive to meropenem/imipenem and aminoglycoside only.

5.Sensitive to cephalosporins only.

6.Resistant to aminoglycosides only.

We chose a meripenum/imepenum and aminoglycosides sensitive baumanii isolate for strain-to-reference sequencing on an Illumina Genome Analyzer II system at the Vanderbilt University Genome Technology Core (https://gtc.vanderbilt.edu/gtc/tech).

\section{Conclusion}

Initial sequencing yielded 5,250,420 reads of 43bp each, yielding $225.76 \mathrm{Mb}$ of total sequence. The reads from our isolate were aligned to MDR baumannii reference strain ACICU (NC_010611.1). Alignment was done with the Bowtie Aligner [7]. Of the 5.2 million total reads, $4,004,012(76.26 \%)$ aligned to AICIU, with a mean coverage depth of 43.96 fold. Roughly $58 \%$ of the ACICU genome was covered by at least one read. We will next align the reads further with other baumannii reference strains including MDR AYE (NC_010410) and nonresistant strain SDF (NC_010400) in order to further characterize and annotate our isolate at the genomic level.

\section{Acknowledgements}

Experiment design and data analysis performed through the use of the Meharry Medical College Microarray and Bioinformatics Core, which is supported in part by NIH grants G12RR03032-19 and P20RR011792. (http:// www.mmc.edu/bioinformatics/)

Sequencing and alignment was performed at the Vanderbilt University Genome Technology Core (https://gtc.vanderbilt.edu/gtc/tech).

\section{Author details}

'Department of Surgery, Meharry Medical College, Nashville, TN 37208, USA. ${ }^{2}$ Nashville General Hospital, Nashville, TN 37208, USA. ${ }^{3}$ Microarray and 


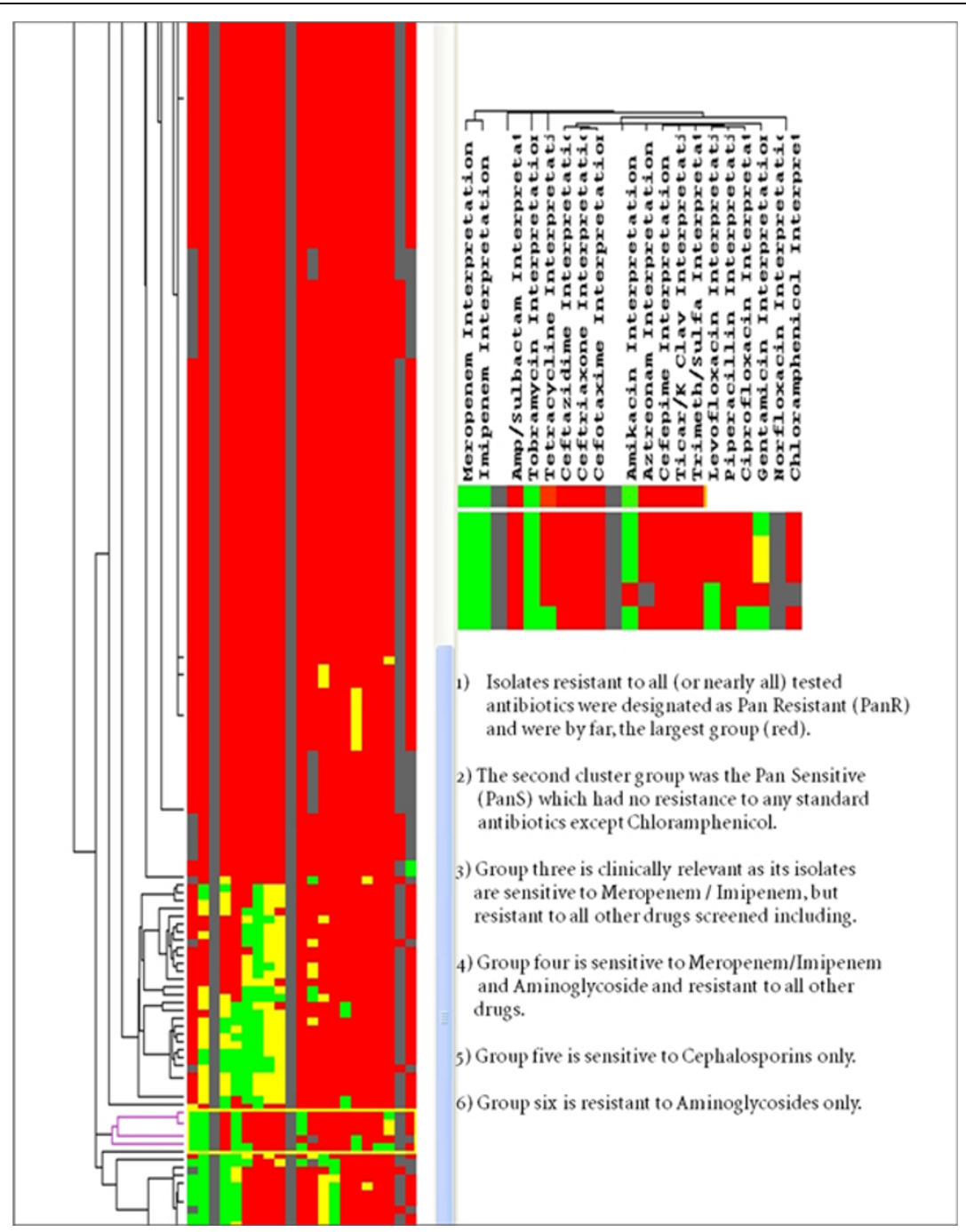

Figure 1 Clustered baumannii groups. Left Side: full clustergram of all isolate phenotypes. Right Side: zoom in of resistance group 4: meripenem/imipenum and aminoglycoside sensitive. Legend: red = resistant, green = sensitive, yellow = intermediate, black/gray = no data.

Bioinformatics Core, Dept. of Microbiology, Meharry Medical College, Nashville, TN 37208, USA.

Published: 23 July 2010

\section{References}

1. Garcia-Garmendia J, Ortiz-Leyba C, Garmacho-Montero J, JimenezJimenez FJ, Monterrubio-Villar J, Gili-Miner M: Mortality and the increase in length of stay attributable to the acquisition of Acinetobacter in critically ill patients. Crit Care Med 1999, 27(9):1794-1799.

2. Falagas ME, Rafailidis PI: Attributable mortality of Acinetobacter baumannii: no longer a controversial issue. Critical Care (London, England) 2007, 11(3):134.

3. Jamulitrat $S$, Arunpan $P$, Phainuphong $P$ : Attributable mortality of imipenem-resistant nosocomial Acinetobacter baumannii bloodstream infection. J Med Assoc Thai 2009, 92(3):413-419.

4. Bonomo RA, Szabo D: Mechanisms of multidrug resistance in Acinetobacter species and Pseudomonas aeruginosa. Clin Infect Dis 2006, 43(Suppl 2):S49-S56.

5. Fournier PE, Vallenet $D$, Barbe V, Audic S, Ogata $H$, Poirel L, Richet $H$, Robert C, Mangenot S, Abergel C, Nordmann P, Weissenbach J, Raoult D,
Claverie JM: Comparative genomics of multidrug resistance in Acinetobacter baumannii. Plos Genet 2006, 2(1):e7.

6. Eisen MB, Spellman PT, Brown PO, Botstein D: Cluster analysis and display of genome-wide expression patterns. PNAS 1998, 95:14863-14868.

7. Langmead B, Trapnell C, Pop M, Salzberg SL: Ultrafast and memoryefficient alignment of short DNA sequences to the human genome. Genome Biol 2009, 10:R25, doi:10.1186/gb-2009-10-3-r25.

doi:10.1186/1471-2105-11-S4-P16

Cite this article as: Dent et al:: Next-gen sequencing of multi-drug resistant Acinetobacter baumannii to determine antibiotic resistance genotypes. BMC Bioinformatics 2010 11(Suppl 4):P16. 